\title{
Special issue on agreement technologies
}

\author{
Carlos Iván Chesñevar ${ }^{1} \cdot$ Eva Onaindia $^{2} \cdot$ Sascha Ossowski $^{3} \cdot$ George Vouros $^{4}$
}

Published online: 10 July 2015

(C) Springer Science+Business Media New York 2015

Most would agree that large-scale open distributed systems are an area of enormous social and economic potential. In fact, regardless of whether they are realised for the purpose of business or leisure, in a private or a public context, people's transactions and interactions are increasingly mediated by computers. The resulting networks are usually large in scale, involving millions of interactions, and are open for the interacting entities to join or leave at will. We have been experiencing a paradigm shift in the way that such systems are built, enacted, and managed: away from rigid and centralised client-server architectures, towards more flexible and decentralised means of interaction. Nowadays, there is a growing awareness that the notion of agreement and agreement processes will be of key importance for the next generation of such decentralised large-scale open distributed systems (Ossowski et al. 2013).

George Vouros

georgev@unipi.gr

Carlos Iván Chesñevar

cic@cs.uns.edu.ar

Eva Onaindia

onaindia@dsic.upv.es

Sascha Ossowski

sascha.ossowski@urjc.es

1 Department of Computer Science \& Engineering, Universidad Nacional del Sur (UNS), Av. Alem 1253, B8000CPB Bahía Blanca, Argentina

2 Universitat Politecnica de Valencia, Camino de Vera s/n, 46022 Valencia, Spain

3 Centre for Intelligent IT (CETINIA), University Rey Juan Carlos Calle Tulipán s/n, 28933 Móstoles Madrid, Spain

4 Department of Digital Systems, University of Piraeus, 126 Gr. Lambraki Str, 18534 Piraeus, Greece
Until recently, the concept of agreement was a domain of study mainly for philosophers and sociologists and it was applied exclusively to human societies. In recent years, the growth of disciplines such as social psychology, socio-biology, social neuroscience, together with the spectacular emergence of the information society technologies, have changed this situation. Presently, agreement and all the processes and mechanisms related with reaching agreements between different kinds of agents are a subject of research and analysis also from technology-oriented perspectives. Casanovas (2013), for instance, tackles this matter from within the context of philosophy and sociology of law. A study from the perspective of cognitive science and technology is introduced by Paglieri (2013).

In one or another way, the notion of agreement has always been relevant to the development software systems as well. Traditionally, it has been based on implicit agreements between programmers and language designers. Thanks to the formal semantics of programming languages specified by their designers, programmers are aware of the meaning of the computational concepts used to write and execute a program. For instance, programmers who use some imperative programming languages share the meaning of the concept of variable, value or loop as well as the notion of program state and state transition. As software systems become bigger and more complex, more dynamic and explicit agreements between project leaders and programmers are needed, in order to establish and document the relationships between the different software components. These agreements (e.g. in the form of specifications of interface and behaviour) become then the basis for the subsequent implementation and verification of the resulting software product. They are established dynamically at design-time, as project leaders and programmers need to interact and discuss the component specifications. However, once such agreements are reached for all elements of the software system, they necessarily remain unchanged at execution time. But, when 
software systems and their elements become open, adaptive, and autonomic, it turns out to be impossible to explicitly define such agreements at design-time. Some software elements may need to interact with others that their programmer was unaware of at design-time. In addition, they cannot rely on complete functional descriptions of the elements that they interact with, as certain behaviours may vary at run-time in response to changes in the environment. Therefore, agreements need to be forged dynamically at run-time, and there must be mechanisms for re-assessing and revising them as execution progresses (Ossowski et al. 2013).

In this special issue, we focus our attention on large-scale open distributed systems whose interacting elements are software agents. There is still no consensus where to draw the border between programs or objects on the one hand and software agents on the other. Perhaps the most commonly accepted characterisation of the term was introduced by Wooldridge and Jennings (1995) who put forward four key hallmarks of agenthood:

- Autonomy: agents should be able to perform the majority of their problem solving tasks without the direct intervention of humans or other agents, and they should have a degree of control over their own actions and their own internal state.

- Social ability: agents should be able to interact, when they deem appropriate, with other software agents and humans in order to complete their own problem solving and to help others with their activities where appropriate.

- Responsiveness: agents should perceive their environment (which may be the physical world, a user, a collection of agents, the INTERNET, etc.) and respond in a timely fashion to changes which occur in it.

- Proactiveness: agents should not simply act in response to their environment, they should be able to exhibit opportunistic, goal-directed behaviour and take the initiative where appropriate.

In order to show the aforementioned properties, the interactions of the software agent with its environment (and with other agents) must be guided by a reasonably complex program, capable of rather sophisticated activities such as reasoning, learning, or planning.

Agreement Technologies (AT) refer to decentralised computer systems in which software agents negotiate with one another, typically on behalf of humans, in order to come to mutually acceptable agreements. In particular, the vision AT comprises next-generation methods and techniques that support computational agents in open and distributed settings to reach agreements of different kinds: agreements on the semantics of the agents' internal representations, on the ways the agents conceptualize the world, on the data gathered (even from third parties) and on the information they share.
Agreements can also be established on policies/norms so that agents can jointly act safely and rationally in a common environment, or on the ways agents share tasks and roles towards organized joint - coordinated - action; as well as on the way such agents share their assessments on the trustworthiness of others. The following technologies are needed in a sandbox to define, specify and verify such systems.

- Semantic Technologies: In an open and large scale distributed system such as the ones covered by Agreement Technologies, where agents are mostly autonomous, applications, services, communication devices, social entities, etc. are likely to comply with very different data models, knowledge representation, functionalities and so on. Thereby, in order to make them interact appropriately to exchange information and reach common goals, there is a need for agents to carry and offer an explicit semantic interface in such a way that agents can mutually "understand" each other. However, in open systems where agents can enter the system at any time having their own interfaces, we can not assume that they all adhere to a single view of the world. Thus, heterogeneity is a strong obstacle for agents to interoperate properly. Semantic technologies provide good solutions to unlock the barriers towards interoperability.

- Norms: Norms (policies or social conventions) are important in Multi Agent Systems to regulate agents' behaviour and support agents to coordinate effectively, also addressing different aspects of security. Therefore, we can say that norms are important mechanisms, "shaping" agents agreements towards action. However, there are important issues concerning norms' conflicts, violation and revision of norms that are within AT scope. Furthermore, emergence of social conventions/norms are important to open systems, as well as monitoring the adherence to norms, enforcing and measuring the success of norms.

- Multiagent organizations and institutions: Organized behaviour of agents may stem from norms, conventions and policies in a society, but organisations and institutions provide an interesting perspective for Agreement Technologies. These are important to specify how to solve a complex task or problem by a number of agents in a declarative way, emphasizing on agents' roles, teamwork, deontic aspects, organizational structures, tasks and goals; also studying agents' organized adaption. Moreover the need to model and figure out properties of sociotechnical systems, and further advance the implementation of complex systems in terms of institutional aspects (e.g. the enforcement of norms for reaching agreements in open environments), has shown the importance of studying multiagent institutions.

- Argumentation and negotiation: Argumentation provides a powerful mechanism for dealing with incomplete, 
possibly inconsistent information and it is fundamental for the resolution of conflicts and differences of opinion amongst different parties. Agreement also benefits from negotiation, especially when autonomous agents have conflicting interests/desires but may benefit from cooperation in order to achieve them. Typically negotiation involves (fair) compromise. As a consequence, argumentation and negotiation are important to reaching agreements among agents, while their interaction with other agreement technologies are important to realizing the open multiagent systems needed.

- Trust and reputation: Since the appearance of the multiagent systems paradigm, the importance of trust and reputation to regulate the behaviour of agents became clear. Recent advances consider trust and reputation models as highly intertwined parts together with other parts of the agent in the context of the different processes that revolve around the notion of interaction with others as well as with its environment.

As we delve into agreement technologies, we see how these technologies are intertwined: agents can take advantage of semantic agreements, but semantic agreement methods can also take advantage of methods concerning agents' organized action, of trustworthiness and reputation assessments, of advances on multi-agent systems argumentation and negotiation, and of findings concerning (sometimes emergent) normative behaviour. We can see several other dependencies that can be of benefit to computational agents: for instance, agreeing on trustworthiness and on aligning communication protocols towards successful interoperation in multi-agent systems, by exploiting the semantics of trust evaluation and commitments, respectively.

In addition, the wide range of social theories available todate offers many different solutions to problems found in complex (computer) systems. So, deciding which theories to apply - as well as how and when - becomes a major challenge. This kind of interdisciplinary research is necessary to work towards a more robust understanding of the notion of agreement and all the processes and mechanisms involved in reaching agreements between different kinds of agents, thus laying the Agreement Technologies paradigm upon more solid conceptual foundations.

In recent years, researchers from the field of multiagent systems, semantic technologies, as well as social sciences have joined together to work towards that vision. We particularly refer to the products of the Agreement Technologies COST action (IC0801), as well as to the two Agreement Technologies conferences organized in 2012 (AT 2012, Dubrovnik, Croatia) and 2013 (AT 2013, Beijing, China).

This special issue gathers seven articles that report on works that have presented in AT 2013, have considerably revised and extended according to reviewers' comments, discussions and feedback authors received during this long process.

The first paper contributes to understanding a crucial aspect concerning agreements: preferences. In their article on "Reasoning about reasons behind preferences using modal logic", Pedersen et al. (2015) challenge the assumption that preferences are primitive objects, and - in line with recent advances on social choice theory - question how they are formed, where they come from and how they can be exploited during reasoning in multiagent systems towards reaching consensus. Their proposal is a modal logic for reasoning about preferences that depend on "motivationally salient" properties of objects. The main result is a translation showing how reasoning in this logic can be captured by reasoning in a standard modal logic (KT with universal modality). Authors discuss how the proposed approach allows for straightforward generalization to the multiagent case, and how it can allow reasoning about agents who disagree because they are motivated by different factors.

The second paper, entitled "Reasoning about impacts of information sharing" by Bisdikian et al. (2015), propose a decisionmaking framework for agents to decide what information to reveal to its neighbours: Actually, they propose a trust based decision mechanism for assessing the effects of information release to any neighbor. The decisions that agents make are directed towards modifying messages so as to become most beneficial to information producers. These decisions exploit agents' subjective beliefs of others, based on the notion of trust. Authors propose a model for information propagation among agents and specify agents' decision process.

In their article on "Subjective logic operators in trust assessment: an empirical study", Cerutti et al. (2015), through a rigorous empirical study on subjective logic operators that are geometrically interpreted, show that these operators offer advantages over standard subjective logic operators for computational models of trust and reputation, requiring minimal changes to existing systems. Specifically, the authors propose a set of desirable properties that discounting and fusion operators must satisfy and present a set of operators satisfying these properties.

Ramon Hermoso, Henrique Lopes Cardoso and Maria Fasli, propose an approach to the maintenance of roles using incentives. In their article "From roles to standards: a dynamic maintenance" (Hermoso et al. 2015) they deal with the changes on the expectations from roles in dynamically evolving multiagent systems. Using standards capturing the level of suitable performance that agents have to show when they carry out tasks, authors propose an incentive-based mechanism to maintain roles over time. As authors claim, this is in contrast to reorganization in dynamically evolving systems when incentives are not cost-effective.

Argumentation is a powerful means to reach agreements, but not without considering the context in which it happens: 
this is the main conjecture behind the work reported in the article entitled "An ontological-based knowledge-representation formalism for case-based argumentation" by Heras et al. (2015). Specifically, authors propose an ontological-based framework for the design of multiagent systems, in which the agents - participants in a cased-based argumentation framework- can exchange arguments taking into account their social context.

Aiming at an efficient service discovery process, Zijie Cong and Alberto Fernandez in the article "Service discovery acceleration with hierarchical clustering" (Cong and Fernandez 2015) propose an approach in which services are hierarchically clustered using a distance measure computed by a matcher. Authors show that while searching in the resulting tree may result to sacrificing precision at acceptable levels, the service discovery process is dramatically improved.

In the article entitled "On the inaccuracy of numerical ratings: dealing with biased opinions in social networks", Centeno et al. (2015), deal with the subjectivity issues concerning numerical rankings of entities' reputation in social networks. Their proposal concerns gathering comparative opinions about entities, from users. Users and entities to be queried are selected based on their social context and on what authors call the principle of heterogeneity.

We wish to extend our warm thanks to all researchers and practitioners of the Agreement Technologies community, and in particular to the members of COST Action IC0801, and to the delegates of the conferences in the Agreement Technologies series. A special "thank you" goes to AT-2013 authors who submitted their papers for consideration in this special issue, as well as to the AT 2013 Program Committee members and additional reviewers (please refer to the AT 2013 website $^{1}$ for the full list of names), as well as the reviewers of this special issue (mentioned at the end of this editorial) for allowing us to put together this vibrant collection of high-quality papers. We hope you will enjoy reading our special issue on Agreement Technologies.

\section{Special Issue Reviewers}

Jurgen Dix, Clausthal University of Technology, Germany.

Giulia Andrighetto, National Research Council - Institute of Cognitive Sciences and Technologies, Italy.

Estefanía Argente, Universitat Politècnica de València, Spain.

Olivier Boissier, ISCOD/LSTI - Institut Henri Fayol, École Nationale Supérieure des Mines de Saint-Étienne, France.

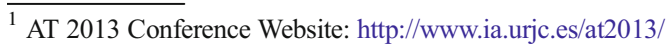

Henrique Lopes Cardozo, University of Porto, Portugal.

Carlos Ivan Chesñevar, Universidad Nacional del Sur, Bahía Blanca, Argentina.

Maria Ganzha, University of Gdansk, Poland.

Mirjana Ivanovic, University of Novi Sad, Serbia.

Lea Kutvonen, University of Helsinki, Finland.

Luis Gustavo Nardin, Institute of Cognitive Sciences and Technologies (ISTC), Italy.

Eugenio Oliveira, University of Porto, Portugal.

Jordi Sabater, Spanish National Research Council - The Artificial Intelligence Research Institute, Spain.

Michael Schumacher, University of Applied Sciences

Western Switzerland, Switzerland.

Laszlo Varga, Hungarian Academy of Sciences - The

Computer and Automation Research Institute, Hungary.

Matteo Vasirani, Ecole Polytechnique Federale de Lausanne, Switzerland.

\section{References}

Bisdikian, C., Cerutti, F., Tang, Y., \& Oren, N. (2015). Reasoning about impacts of information sharing. Information Systems Frontiers, 17(4). doi:10.1007/s10796-014-9521-6.

Casanovas, P. (2013) Agreement and relational justice - a perspective from philosophy and sociology of law. In S. Ossowski et al. (Eds.), Agreement technologies. Law, governance and technologies series (Vol. 8, pp. 17-41). Springer Verlag.

Centeno, R., Hermoso, R., \& Fasli, M. (2015). On the inaccuracy of numerical ratings: dealing with biased opinions in social networks. Information Systems Frontiers, 17(4). doi:10.1007/s10796-0149526-1.

Cerutti, F., Kaplan, L. M., Norman, T. J., Oren, N., \& Toniolo, A. (2015). Subjective logic operators in trust assessment: an empirical study. Information Systems Frontiers, 17(4). doi:10.1007/s10796-0149522-5.

Cong, Z., \& Fernandez, A. (2015). Service discovery acceleration with hierarchical clustering. Information Systems Frontiers, 17(4). doi: 10.1007/s10796-014-9525-2.

Heras, S., Botti, V., \& Julian, V. (2015). An ontological- based knowledgerepresentation formalism for case-based argumentation. Information Systems Frontiers, 17(4). doi:10.1007/s10796-014-9524-3.

Hermoso, R., Lopes Cardoso, H., \& Fasli, M. (2015). From roles to standards: a dynamic maintenance Information Systems Frontiers, 17(4). doi:10.1007/s10796-014-9523-4.

Ossowski, S., et al. (Eds.) (2013). Agreement technologies. Law, governance and technologies series (Vol. 8). Springer Verlag.

Ossowski, S., Sierra, C., Botti, V. (2013) Agreement technologies - a computing perspective. In S. Ossowski et al. (Eds.), Agreement technologies. Law, governance and technologies series (Vol. 8, pp. 3-16). Springer Verlag.

Paglieri, F. (2013) Agreements as the Grease (Not the Glue) of society - a cognitive and social science perspective. In S. Ossowski et al. (Eds.), Agreement technologies. Law, governance and technologies series (Vol. 8, pp. 43-67). Springer Verlag.

Pedersen, T., Dyrkolbotn, S., \& Agotnes, T. (2015). Reasoning about reasons behind preferences using modal logic. Information Systems Frontiers, 17(4). doi:10.1007/s10796-014-9520-7.

Wooldridge, M., \& Jennings, N. (1995). Intelligent agents - theory and practice. Knowledge Engineering Review, 10(2), 115-152. 
Carlos Iván Chesñevar is independent researcher from the National Council of Scientific and Technical Research (CONICET), Argentina, and vice-director of the Research Institute for Computer Science and Engineering of the Universidad Nacional del Sur (ICIC-UNS, Bahía Blanca, Argentina). He has carried out posdoctoral activities at the University of Lleida (Spain) as researcher in Artificial Intelligence from 2003 to 2007 . He has led several scientific projects related to argumentation and technological applications, supported by different funding agencies (DAAD Germany, CONICET Argentina, Microsoft Research Latinamerica, etc.). He has been also representing Argentina as an expert in argumentation within an ESF-funded COST Action on Agreement Technologies. He has participated as PC member in most major AI conferences (IJCAI, AAMAS, ECSQARU, etc.) and has published more than 25 journal articles, 7 book chapters and more than 100 papers in international and national conferences in his research area. He has carried out research stances in different universities (Imperial College, London, UK; University Leipzig, Leipzig, Germany;Washington University, USA, among others). He has supervised several $\mathrm{PhD}$ and MSc Theses in Computer Science, particularly related to argumentation in Artificial Intelligence and its applications. Carlos Chesñevar has also been a member of the ICEGOV Programme Committee for the conferences in 2013 and 2014.

Eva Onaindia is an Associate Professor of Computer Science at the Universitat Politècnica of València. She received her Ph.D. in Computer Science from the same University in 1997. Since 1994, she teaches undergraduate and master courses in Artificial Intelligence and Planning. She currently leads the group of Reasoning on Planning and Scheduling (http://users.dsic.upv.es/grupos/grps/) where she conducts research in classical and temporal planning, negotiation, argumentation and social choice applied to multi-agentplanning. She has led several national research projects as well as sitting on various scientific committees in her field (IJCAI, ICAPS, AAAI, ECAI, etc.). She is Editor-in-Chief of the journal AI Communications and a Review Board member of the journal Applied Intelligence. She has published about 120 articles in specialized conferences and scientific journals related to topics of Artificial Intelligence, Planning, Argumentation and Recommender Systems. She is also currently undertaking investigations on modeling emotions in decision-making.

Sascha Ossowski is a Full Professor of Computer Science and the Director of the Centre for Intelligent Information Technologies (CETINIA) at
University Rey Juan Carlos in Madrid. Formerly, he was an HCM/TMR research fellow at the AI Department of Technical University of Madrid. Prof. Ossowski has been principal investigator on numerous research grants and contracts in the field of advanced software systems, funded by the regional, national and European bodies. He has authored more than 200 research papers, focusing on the application of Artificial Intelligence techniques to real world problems in domains such as m-Health, Transportation Management, or Smart Grids. Recently, he has been particularly active in the field of Multiagent Systems and the Semantic Web. He is coeditor of more than 20 books, proceedings, and special issues of international journals. He belongs to the board of directors of the International Foundation for Autonomous Agents and Multiagent Systems (IFAAMAS), and is a member of the steering committee of the ACM Annual Symposium on Applied Computing (SAC). He has been the chair of COST Action IC0801 on Agreement Technologies, and was cofounder and first chair of the board of directors of the European Association for Multiagent Systems (EURAMAS). He also serves as a member of the editorial board for several international journals, and acts as programme committee member for numerous internationalconferences and workshops.

George Vouros is Full Professor at the Department of Digital Systems, ICT School, University of Piraeus; head of the Artificial Intelligence Laboratory (http://ai-group.ds.unipi.gr) and member of the Data Science Lab (www.datastories.org). Born in 1964, he holds BSc in Mathemetics and Ph.D. in Computer Science (Artificial Intelligence), both from the National Kapodistrian University of Athens, Greece. His research interests include data integration and interoperability, ontologies and Multiagent Systems, whereas he teaches Semantic Web, Multiagent Systems and Artificial Intelligence at under- and post-graduate level. $\mathrm{He}$ is or was principal investigator / senior researcher for a number of EUfunded and National research projects. He has served and serves as cochair of international conferences and workshops (Agreement Technologies, WI, SETN, COIN, EUMAS), member of steering committee of COIN and SETN, PC member for numerous conferences, including ECAI, AAMAS, RULEML, WWW, Web Intelligence, ICTAI. He serves as reviewer in well-respected journals for data and knowledge engineering, semantic web and multiagent systems. He has delivered invited lectures and seminars on semantic agreements and multi agent systems. He has co-edited post-proceedings and special issues in topics of his research interests and have published more than 150 refereed articles in scientific journals and conferences. 